\title{
Sheared-Flow Modes in Toroidal Geometry
}

\author{
J.L.V. Lewandowski, Z. Lin, W.W. Lee and T.S. Hahm \\ Princeton Plasma Physics Laboratory, \\ Princeton University, P.O. Box 451, Princeton NJ 08543
}

(October 12, 1999)

\begin{abstract}
Using a Fourier-Bessel representation for the fluctuating (turbulent) electrostatic potential, an equation governing the sheared-flow modes in toroidal geometry is derived from the gyrokinetic Poisson equation, where both the adiabatic and non-adiabatic responses of the electrons are taken into account. It is shown that the principal geometrical effect on sheared-flow modes of the electrostatic potential is due to the flux-surface average of $1 / B$, where $B$ is the magnetic field strength.
\end{abstract}

Pacs \# : 52.35Kt, 52.30Jb, 52.35Ra 


\section{INTRODUCTION}

It is now generally accepted in the fusion community that low-frequency, small-scale instabilities (eg. drift waves, Ion Temperature Gradient-driven (ITG) modes) are major contenders for the anomalous, cross-field transport ${ }^{1,2}$ observed in tokamaks ${ }^{3}$ and stellarators $^{4}$. There is some experimental evidence ${ }^{5}$ that (equilibrium) sheared flows (or zonal flows $^{6}$ ) can have a strong impact on the turbulence-driven cross-field transport ${ }^{7}$. Therefore an accurate calculation of sheared-flow modes in toroidal geometry is an important issue.

In this paper, sheared-flow modes in toroidal geometry are calculated from the gyrokinetic Poisson's (GKP) equation ${ }^{8}$. A change of coordinate system allows us to write the fluctuating electrostatic potential in terms of Bessel-Fourier series. The case of a cylindrical plasma has been considered by Li, Lee and Parker. In an unpublished report, these authors discuss the solution of the full GKP in cylindrical geometry with circular magnetic surfaces and no azimuthal magnetic field. By introducing a coordinate transformation, we extend the work of $\mathrm{Li}$ and co-workers to toroidal geometry; furthermore, the main goal of this paper is to consider the calculation of sheared-flow modes in toroidal geometry, rather than the solution of the (full) GKP in toroidal geometry, which requires a numerical approach ${ }^{9}$. It is shown that the principal geometrical effect on sheared-flow modes is due to the flux-surface average of the inverse of the magnetic field strength. The paper is organized as follows; in section II, we introduce the gyrokinetic Poisson's equation; magnetic, toroidal (Shafranov-like) and cylindrical coordinate systems are discussed and the tranformation between guiding center and particle coordinates are given in section III; in the same section, the flux-surface average of the GKP equation is derived and an equation governing the sheared-flow modes in toroidal geometry is obtained; we conclude with some remarks in section IV. 


\section{GYRO-KINETIC POISSON EQUATION}

Assuming that the Debye length is much smaller than the ion thermal gyroradius and neglecting electron gyroradius effects, the GKP equation can be written as ${ }^{8,10,11}$

$$
\int d \mathbf{R}_{\mathrm{gc}} \int d \mathbf{v}\left(F_{\mathrm{i}}+\frac{e \tilde{\Phi}}{m_{\mathbf{i}} B} \frac{\partial F_{\mathbf{i}}}{\partial \mu}\right) \delta\left(\mathbf{R}_{\mathrm{gc}}-\mathbf{r}+\boldsymbol{\rho}\right)=n_{\mathrm{e}},
$$

where $\tilde{\Phi} \equiv \Phi(\mathbf{r})-\langle\Phi\rangle_{\mathrm{gc}}\left(\mathbf{R}_{\mathrm{gc}}, \mathbf{v}\right), \mathbf{r}=\mathbf{R}_{\mathrm{gc}}+\boldsymbol{\rho}$ is the particle position, $\mathbf{R}_{\mathrm{gc}}$ is the guiding center position, $\langle\ldots\rangle_{\mathrm{gc}}$ denotes a gyrophase average keeping the guiding center position fixed, $\boldsymbol{\rho} \equiv \hat{\mathbf{e}}_{\|} \times \mathbf{v} / \omega_{\mathrm{c}}$ is the ion gyroradius, $\widehat{\mathbf{e}}_{\|} \equiv \mathbf{B} / B$ is the unit vector along the confining magnetic field, $\omega_{\mathrm{c}}$ is the ion cyclotron frequency, $\mu=v_{\perp}^{2} /(2 B)$ is the magnetic moment, $F_{\mathrm{i}}$ is the (total) ion guiding center distribution and $n_{\mathrm{e}}$ is the electron density. The aim of this paper is to calculate the flux-surface-averaged component of the electrostatic potential, $\langle\Phi\rangle_{S}$, from the GKP equation (1) in toroidal geometry. The operation $\int d \mathbf{R}_{\mathrm{gc}}[\ldots] \delta\left(\mathbf{R}_{\mathrm{gc}}-\mathbf{r}+\boldsymbol{\rho}\right)$ acts to transform the guiding center variables $\left\{\mathbf{R}_{\mathrm{gc}}, \mathbf{v}\right\}$ to the particle variables $\{\mathbf{r}, \mathbf{v}\}$. Although $F_{\mathrm{i}},\langle\Phi\rangle_{\mathrm{gc}}$ and $B$ are functions of the guiding center variables, the integrand in equation (1) also depends on the particle variable $\{\mathbf{r}, \mathbf{v}\}$ through $\Phi$. We write the ion distribution function as $F_{\mathrm{i}}=F_{\mathrm{i} 0}($ equilibrium $)+\delta \mathrm{F}_{\mathrm{i}}$ (fluctuation) and assume that the equilibrium part of $F_{\mathrm{i}}$ is Maxwellian

$$
F_{\mathrm{i} 0}=\frac{n_{0}\left(\mathbf{R}_{\mathrm{gc}}\right)}{2 \pi v_{\mathrm{thi}}^{2}} \exp \left(-\mu B / v_{\mathrm{thi}}^{2}\right) f\left(\mathbf{R}_{\mathrm{gc}}, v_{\|}\right),
$$

where $v_{\text {thi }} \equiv\left(T_{\mathrm{i} 0}\left(\mathbf{R}_{\mathrm{gc}}\right) / m_{\mathbf{i}}\right)^{1 / 2}$ is the ion thermal velocity and $f$ satisfies the normalization

condition $\int_{-\infty}^{+\infty} d v_{\|} f\left(\mathbf{R}_{\mathrm{gc}}, v_{\|}\right)=1$. The volume element in velocity space is defined as $d^{3} \mathbf{v} \equiv 2 \pi v_{\perp} d v_{\perp} d v_{\|}$. Assuming that $\left|\partial \delta F_{\mathrm{i}} / \partial \mu\right| \ll\left|\partial F_{\mathrm{i} 0} / \partial \mu\right|$ and using $F_{\mathrm{i} 0}\left(\mathbf{R}_{\mathrm{gc}}\right) \approx F_{\mathrm{i} 0}(\mathbf{r})$ and $T_{\mathrm{i} 0}\left(\mathbf{R}_{\mathrm{gc}}\right) \approx T_{\mathrm{i} 0}(\mathbf{r})$, carrying out the integration over $v_{\|}$in equation (1), the GKP equation becomes

$$
\int_{0}^{\infty}\langle\tilde{\Phi}\rangle_{\mathrm{part}} V_{\perp} \exp \left(-V_{\perp}^{2} / 2\right) d V_{\perp}=\frac{T_{\mathrm{i} 0}(\mathbf{r})}{e n_{0}(\mathbf{r})}\left(\overline{n_{\mathbf{i}}}-n_{\mathrm{e}}\right),
$$

where $V_{\perp} \equiv v_{\perp} / v_{\text {thi }}$ is the normalized perpendicular velocity, $\langle\ldots\rangle_{\text {part }}$ denotes a gyrophase angle keeping the particle position fixed and 


$$
\overline{n_{\mathbf{i}}} \equiv \int d \mathbf{R}_{\mathrm{gc}} \int d \mathbf{v} F_{\mathrm{i}}\left(\mathbf{R}_{\mathrm{gc}}, \mathbf{v}\right) \delta\left(\mathbf{R}_{\mathrm{gc}}-\mathbf{r}+\boldsymbol{\rho}\right)
$$

can be calculated numerically by keeping the guiding center position fixed. The electron density can be written as $n_{\mathrm{e}}=n_{\mathrm{e}}^{(\mathrm{A})}+n_{\mathrm{e}}^{(\mathrm{NA})}$, where $n_{\mathrm{e}}^{(\mathrm{A})}$ and $n_{\mathrm{e}}^{(\mathrm{NA})}$ are, respectively, the adiabatic and non-adiabatic parts of the electron density. The adiabatic response if of the form $n_{\mathrm{e}}^{(\mathrm{A})} \propto \Phi-\langle\Phi\rangle_{S}$, where $\langle\ldots\rangle_{S}$ denotes an average over the magnetic surface (see next section).

\section{MAGNETIC AND TOROIDAL COORDINATES}

We consider a fully 3-dimensional configuration with closed, nested magnetic surfaces. The confining magnetic field $\mathbf{B}$ is written in straight-field line coordinates $\{\bar{\rho}, \bar{\theta}, \bar{\zeta}\}$ as

$$
\mathbf{B}=\nabla \alpha \times \nabla \Psi
$$

where $\alpha \equiv \bar{\zeta}-q \bar{\theta}$ is the field line label, $2 \pi \Psi$ is the enclosed poloidal flux, $\bar{\theta}$ is the magnetic poloidal angle and $\bar{\zeta}$ is the magnetic toroidal angle. The radial label is denoted $\bar{\rho}$ and, by definition, $\mathbf{B} \cdot \nabla \bar{\rho} \equiv 0$. There is some freedom in specifying the radial label; here, for

convenience, we define $\bar{\rho} \equiv \sqrt{\psi_{\mathrm{T}} / \psi_{\mathrm{T}}^{(\mathrm{b})}}$, where $\psi_{\mathrm{T}}$ is the toroidal flux enclosed within the magnetic surface, and $\psi_{\mathrm{T}}^{(\mathrm{b})}$ is $\psi_{\mathrm{T}}$ evaluated at the plasma boundary. By construction the radial label runs from 0 (magnetic axis) to 1 (last closed magnetic surface). The magnetic surfaces can be specified in cylindrical coordinates; for instance, in the equilibrium code VMEC $^{12,13}$ an expansion in Fourier series is used

$$
\begin{aligned}
& R_{l}=R_{l}(\bar{\rho}, \bar{\theta}, \bar{\zeta})=\sum_{M=0}^{M_{\max }} \sum_{N=-N_{\max }}^{+N_{\max }} R_{M N} \cos \left(\Theta_{M N}\right) \\
& Z_{l}=Z_{l}(\bar{\rho}, \bar{\theta}, \bar{\zeta})=\sum_{M=0}^{M_{\max }} \sum_{N=-N_{\max }}^{+N_{\max }} Z_{\operatorname{mn}} \sin \left(\Theta_{M N}\right) \\
& \phi_{l}=\phi_{l}(\bar{\rho}, \bar{\theta}, \bar{\zeta})=\zeta-\frac{2 \pi}{N_{\mathrm{per}}} \sum_{M=0}^{M_{\max }} \sum_{M=-N_{\max }}^{+N_{\max }} \widetilde{\phi}_{M N} \sin \left(\Theta_{M N}\right)
\end{aligned}
$$

where $\Theta_{M N} \equiv M \bar{\theta}+N_{\text {per }} N \bar{\zeta}$ and $N_{\text {per }}$ is the number of field periods of the configuration. For intermediate analytical calculations, it is convenient to work with the local toroidal 
coordinate system $\left\{r_{L}, \theta_{L}, \phi_{L}\right\}$ shown in Fig 1 . In the plane $\phi_{L}=$ const, the cylindrical radius $R_{L}$ and the height $Z_{L}$ are

$$
\begin{aligned}
& R_{L}=R_{0}+r_{L} \cos \theta_{L} \\
& Z_{L}=r_{L} \sin \theta_{L} .
\end{aligned}
$$

Here $R_{0}$ is the average radius of the magnetic axis defined as

$$
R_{0} \equiv \frac{1}{4 \pi^{2}} \int_{0}^{2 \pi} d \bar{\theta} \int_{0}^{2 \pi} d \bar{\zeta} R_{L}(\bar{\rho}=0, \bar{\theta}, \bar{\zeta})
$$

This definition is convenient for configurations with helical magnetic axis (e.g. heliac-type configurations). The curve $r_{L}=$ const describes a circle in the plane $\phi=$ const. Note that in general $\nabla \bar{\rho} \times \nabla r_{L} \neq 0$, so that $\mathbf{B} \cdot \nabla r_{L} \neq 0$ along the $r_{L}=$ const curve. Using equations (6), one can invert equations (7) to get

$$
\tan \theta_{L}(\bar{\rho}, \bar{\theta}, \bar{\zeta})=\frac{Z_{L}(\bar{\rho}, \bar{\theta}, \bar{\zeta})}{R_{L}(\bar{\rho}, \bar{\theta}, \bar{\zeta})-R_{0}}
$$

and

$$
r_{L}(\bar{\rho}, \bar{\theta}, \bar{\zeta})=\left[\left[R_{L}(\bar{\rho}, \bar{\theta}, \bar{\zeta})-R_{0}\right]^{2}+Z_{L}^{2}(\bar{\rho}, \bar{\theta}, \bar{\zeta})\right]^{1 / 2}
$$

where the explicit dependence on the magnetic coordinates $\{\bar{\rho}, \bar{\theta}, \bar{\zeta}\}$ is shown. Although the inverse transformations $(9,10)$ are exact, the fact that, in general, $\nabla \bar{\rho} \times \nabla r_{L} \neq 0$, complicates the representation of the fluctuating electrostatic potential $\Phi$ in the gyrokinetic Poisson equation. As we shall see below, it is convenient to introduce a flux surfaceaveraged toroidal system $\{r, \theta, \phi\}$. The radial label is defined as $r \equiv\left\langle r_{L}(\bar{\rho}, \bar{\theta}, \bar{\zeta})\right\rangle_{S} / a$ where $a \equiv\left\langle r_{L}(\bar{\rho}=1, \bar{\theta}, \bar{\zeta})\right\rangle_{S}$ and $\langle\ldots\rangle_{S}$ denotes an average over the magnetic surface. For an arbitrary function $F,\langle F\rangle_{S}$ is defined as

$$
\langle F\rangle_{S}(\bar{\rho}) \equiv \frac{1}{A(\bar{\rho})} \int_{0}^{2 \pi} d \bar{\zeta} \int_{0}^{2 \pi} d \bar{\theta} \mathcal{J}(\bar{\rho}, \bar{\theta}, \bar{\zeta}) F(\bar{\rho}, \bar{\theta}, \bar{\zeta})
$$

where $\mathcal{J} \equiv[\nabla \bar{\rho} \cdot(\nabla \bar{\theta} \times \nabla \bar{\zeta})]^{-1}$ is the Jacobian of the transformation and

$$
A \equiv \int_{0}^{2 \pi} d \bar{\zeta} \int_{0}^{2 \pi} d \bar{\theta} \mathcal{J}(\bar{\rho}, \bar{\theta}, \bar{\zeta})
$$


is the area of the magnetic surface $\bar{\rho}=$ const. For a configuration with a single magnetic axis, the relation $r=r(\bar{\rho})$ is monotonous in $\bar{\rho}$ and it can be easily inverted. Then we may write the fluctuating electrostatic potential as

$$
\Phi=\sum_{l=1}^{\infty} \sum_{m, n=-\infty}^{\infty} \Phi_{l m n} J_{m}\left(\beta_{m l} r\right) \exp [i(m \theta+n \phi)]
$$

where $\beta_{m l}$ is the $l^{\text {th }}$ zero of the Bessel function of order $m$. We note that, locallly, $\theta$ and $\phi$ in the above equation are dependent on the magnetic coordinates $\{\bar{\rho}, \bar{\theta}, \bar{\zeta}\}$. Multiplying equation (13) by $r J_{m}\left(\beta_{m l} r\right) \exp (-i m \theta-i n \phi)$ and integrating over $r, \theta$ and $\phi$ yields the coefficients $\Phi_{l m n}$

$$
\Phi_{l m n}=\frac{1}{2 \pi^{2} J_{m+1}^{2}\left(\beta_{m l}\right)} \int_{0}^{1} d r \int_{0}^{2 \pi} d \theta \int_{0}^{2 \pi} r J_{m}\left(\beta_{m l} r\right) \exp (-i m \theta-i n \phi) \Phi(r, \theta, \phi) d \phi
$$

where we have used the relation of orthogonality ${ }^{15}$ of Bessel functions

$$
\int_{0}^{1} x J_{N}(\alpha x) J_{N}(\beta x) d x=\frac{\delta(\alpha-\beta)}{2}\left[J_{N+1}(\alpha)\right]^{2}
$$

The difficulty in calculating the flux-surface average of the GKP equation (1) arises in the $\left\langle\langle\Phi\rangle_{\mathrm{gc}}\right\rangle_{\mathrm{part}}$ term. In particular we must transform back and forth between particle and guiding center variables; it is then convenient to use the cylindrical coordinates $\{R, Z, \phi\}$ and the local toroidal coordinates $\{r, \theta, \phi\}$ (right-handed in that order). It is easy to show the unit vectors in the local toroidal coordinates can be written as

$$
\begin{aligned}
& \widehat{\mathbf{r}}=\cos \theta \widehat{\mathbf{R}}+\sin \theta \hat{\mathbf{Z}}=+\cos \theta \cos \phi \widehat{\mathbf{x}}-\cos \theta \sin \phi \hat{\mathbf{y}}+\sin \theta \hat{\mathbf{z}} \\
& \hat{\boldsymbol{\theta}}=\cos \theta \hat{\mathbf{Z}}-\sin \theta \widehat{\mathbf{R}}=-\sin \theta \cos \phi \hat{\mathbf{x}}+\sin \theta \sin \phi \hat{\mathbf{y}}+\cos \theta \hat{\mathbf{z}} \\
& \widehat{\boldsymbol{\phi}}=-\sin \phi \hat{\mathbf{x}}-\cos \phi \hat{\mathbf{y}} .
\end{aligned}
$$

Then one can use the set $\left(r_{0}, \theta_{0}, \phi_{0}\right)$ to label the guiding center position, and the particle position $\mathbf{r}(r, \theta, \phi)=\mathbf{R}_{\mathrm{gc}}\left(r_{0}, \theta_{0}, \phi_{0}\right)+\boldsymbol{\rho}$ can also be written as (Fig 2$)$

$$
R_{0} \widehat{\mathbf{R}}+r \widehat{\mathbf{r}}=R_{0} \widehat{\mathbf{R}}_{\mathrm{gc}}+r_{0} \widehat{\mathbf{r}}_{0}+\boldsymbol{\rho}
$$

where $\widehat{\mathbf{R}}_{\mathrm{gc}} \equiv \cos \phi_{0} \widehat{\mathbf{x}}-\sin \phi_{0} \hat{\mathbf{y}}$. Noting that the particle velocity can be written as (Fig 3 ) 


$$
\mathbf{v}=v_{\|} \widehat{\mathbf{e}}_{\|}+v_{\perp}(\cos \varphi \hat{\mathbf{n}}+\sin \varphi \hat{\mathbf{b}})
$$

where $\hat{\mathbf{n}} \equiv\left[\nabla \Psi /(\nabla \Psi \cdot \nabla \Psi)^{1 / 2}\right]_{r_{0}, \theta_{0}, \zeta_{0}}$ is the unit normal vector, $\hat{\mathbf{e}}_{\|} \equiv \mathbf{B} / B$ is the unit vector along $\mathbf{B}, \hat{\mathbf{b}} \equiv\left[\widehat{\mathbf{e}}_{\|} \times \widehat{\mathbf{n}}\right]_{r_{0}, \theta_{0}, \zeta_{0}}$ is the unit binormal vector, and $\varphi$ is the gyroangle, the components of equation (17) yield [neglecting corrections $\mathcal{O}\left(\rho^{2} / R_{0}^{2}\right)$ and higher]

$$
\Delta \phi \approx \frac{\rho \cdot \hat{\phi}_{0}}{R},
$$

where, as before, $R=R_{0}+r \cos \theta$ is the cylindrical radius; and

$$
\begin{aligned}
& r \cos \Delta \theta-\rho^{\prime \prime} \cos \psi^{\prime \prime}=r_{0} \\
& r \sin \Delta \theta-\rho^{\prime \prime} \sin \psi^{\prime \prime}=0 .
\end{aligned}
$$

where we have introduce the new variables $\rho "$ and $\psi^{\prime \prime}$ such that

$$
\begin{aligned}
& \rho^{\prime \prime} \sin \psi^{\prime \prime}=\rho \sin \psi\left(\hat{\mathbf{b}} \cdot \hat{\boldsymbol{\theta}}_{0}\right)+\rho \cos \psi\left(\widehat{\mathbf{n}} \cdot \hat{\boldsymbol{\theta}}_{0}\right) \\
& \rho^{\prime \prime} \cos \psi^{\prime \prime}=\rho \cos \psi\left(\widehat{\mathbf{n}} \cdot \hat{\mathbf{r}}_{0}\right)+\rho \sin \psi\left(\widehat{\mathbf{b}} \cdot \hat{\mathbf{r}}_{0}\right)
\end{aligned}
$$

and $\psi \equiv \varphi-3 \pi / 2$, Using the Fourier-Bessel representation (13) and taking into account equations $(19,20,21)$ on can show that (appendix A)

$$
\begin{array}{r}
\left\langle\langle\Phi\rangle_{\mathrm{gc}}\right\rangle_{\mathrm{part}}=\sum_{l=1}^{\infty} \sum_{m, n=-\infty}^{\infty} \Phi_{l m n} \exp (i m \theta+i n \phi)\left\{\sum_{k=-\infty}^{\infty}(-1)^{k} J_{k}\left(\beta_{m l} \rho^{\prime \prime}\right) J_{k}\left(k_{\mathrm{t}} \rho^{\prime \prime}\right)\right. \\
\quad \times J_{m+k}\left(\beta_{m l} r\right) J_{0}\left(\hat{k} \rho^{\prime \prime}\right)+\frac{\beta_{m l}}{4} \rho^{\prime \prime} \sum_{k=-\infty}^{\infty}(-1)^{k} J_{k}\left(\beta_{m l} \rho^{\prime \prime}\right) J_{k}\left(k_{\mathrm{t}} \rho^{\prime \prime}\right) \\
\left.\times\left[J_{m+k+1}\left(\beta_{m l} r\right)-J_{m+k-1}\left(\beta_{m l} r\right)\right]\left[J_{1}\left(x_{+}\right)-J_{1}\left(x_{-}\right)\right]\right\} .
\end{array}
$$

Here $k_{\mathrm{t}} \equiv n g / R$, where $g \equiv\left(\widehat{\mathbf{b}} \cdot \widehat{\phi}_{0}\right) /\left(\widehat{\mathbf{b}} \cdot \hat{\boldsymbol{\theta}}_{0}\right)$, is the toroidal wavenumber; $k_{\mathrm{p}} \equiv m / r$ is the poloidal wavenumber; $\hat{k} \equiv k_{\mathrm{p}}+k_{\mathrm{t}}$ and $x_{ \pm} \equiv(\hat{k} \pm 1 / r) \rho^{\prime \prime}$. In the limit $B_{\theta} \mapsto 0$, it can be shown (appendix B) that equation (22) reduces to

$$
\left\langle\langle\Phi\rangle_{\mathrm{gc}}\right\rangle_{\mathrm{part}}=\sum_{l=1}^{\infty} \sum_{m, n=-\infty}^{\infty} \Phi_{l m n} \exp (i m \theta+i n \phi) J_{m}\left(\beta_{m l} r\right) J_{0}{ }^{2}\left(\beta_{m l} \rho\right)
$$

which is the expression derived by Li et al. Unlike the cylindrical case, we note that $\left\langle\langle\Phi\rangle_{\mathrm{gc}}\right\rangle_{\text {part }}$ depends explicitely on the poloidal and toroidal wavenumbers. Substituting 
$\Phi$ [equation (13)] and $\left\langle\langle\Phi\rangle_{\mathrm{gc}}\right\rangle_{\text {part }}[$ equation (22)] in the GKP equation (3), using the integral representation of the Bessel functions ${ }^{14}$ and carrying out the integration over $V_{\perp}$ yields (appendix B)

$\sum_{l=1}^{\infty} \Phi_{l 00}\left\{J_{0}\left(\beta_{0 l} r\right)\left[1-\exp \left(-C_{l}^{2} / 2\right)\right]+\frac{\left(\left\langle\rho_{\mathrm{i}}\right\rangle_{S} / r\right)}{\Gamma(2)} J_{1}\left(\beta_{0 l} r\right) C_{l}\left(1-\frac{C_{l}^{2}}{2}\right) \exp \left(-C_{l}^{2} / 2\right)\right\}=\mathcal{D}(r)$

where $C_{l} \equiv \beta_{0 l}\left\langle\rho_{\mathbf{i}}\right\rangle_{S}$; here $\left\langle\rho_{\mathbf{i}}\right\rangle_{S}$ is the flux surface average of the ion thermal gyroradius and the right-hand side, $\mathcal{D}(r) \equiv T_{\mathrm{i} 0}\left[\left\langle\overline{n_{\mathbf{i}}}\right\rangle_{S}-\left\langle n_{\mathrm{e}}^{(\mathrm{NA})}\right\rangle_{S}\right] /\left(e n_{0}\right)$, can be evaluated numerically. In deriving the expression for $\mathcal{D}(r)$ we have taken into account the fact that $n_{\mathrm{e}}^{(\mathrm{A})} \propto \Phi-\langle\Phi\rangle_{S}$ so that $\left\langle n_{\mathrm{e}}^{(\mathrm{A})}\right\rangle_{S} \equiv 0$. In equation $(24)$, corrections $\mathcal{O}\left[\left(\left\langle\rho_{\mathbf{i}}\right\rangle_{S} / r\right)^{2}\right] \ll 1$ and higher have been neglected. Since $\left\langle\rho_{\mathbf{i}}\right\rangle_{S} \propto\langle 1 / B\rangle_{S}$, it is clear from equation (24) than the principal geometrical effect on sheared-flow modes is due to the flux-surface average of $1 / B$. However this geometrical dependence is nonlinear. Finally it is worth pointing out that the coefficients of $\Phi_{l 00}$ tend asymptotically towards zero as $C_{l} \mapsto 0$, as it should be; the limit $C_{l} \mapsto 0$ (which is the counterpart of $k_{\perp} \rho_{\mathbf{i}} \mapsto 0$ in slab geometry) correspond to modes that cannot have a radial structure. If higher-order corrections were to be taken into account, the coupling between the $\Phi_{l \pm 10}$ modes and flux-surface averaged equilibrium quantities should be retained in equation (24). Therefore the calculation of sheared-flow modes in low-aspect-ratio configurations (e.g. spherical tokamaks) would require a different approach than presented in this paper.

There are various mechanisms that can excite sheared-flow modes in toroidal geometry. For instance, it has been shown by Diamond and Kim that the poloidal flow in a toroidal plasma can be accelerated when there is a gradient in the turbulent Reynolds stress ${ }^{16}$. As shown by Diamond et $a t^{k}$, zonal flows can be generated by modulational instability of a drift-wave turbulence and, in turn, regulate the turbulence. Furthermore any mechanism that can influence the fraction of non-adiabatic electrons can also modify the sheared-flow modes, through a modification of the 'source term' on the right-hand side of equation (24); the relative importance of the non-adiabatic electrons is of course model dependent. 


\section{CONCLUDING REMARKS}

In this paper, an equation governing sheared-flow modes in toroidal geometry has been derived. By introducing a local system of Shafranov-like toroidal coordinates and by using a Fourier-Bessel representation (which exploits the toroidicity of the configuration) for the electrostatic potential, the flux-surface average of the gyrokinetic Poisson has been obtained. It has been shown that the principal geometrical effect on sheared-flow modes is due to $\langle 1 / B\rangle_{S}$, where $\langle\ldots\rangle_{S}$. The equation for sheared-flow modes (24) can be solved numerically by quadrature. 


\section{APPENDIX A: GYRO-PHASE-AVERAGED ELECTROSTATIC POTENTIAL IN TOROIDAL GEOMETRY}

The basic coordinate systems used in this Appendix are the cylindrical coordinates $\{R, Z, \phi\}$ and the local toroidal coordinates $\{r, \theta, \phi\}$. The unit vectors in cylindrical coordinates can be decomposed onto a local Cartesian system: $\widehat{\mathbf{R}}=\cos \phi \hat{\mathbf{x}}-\sin \phi \hat{\mathbf{y}}$, $\widehat{\boldsymbol{\phi}}=-\sin \phi \hat{\mathbf{x}}-\cos \phi \hat{\mathbf{y}}$ and $\hat{\mathbf{z}}=\hat{\mathbf{z}}$. The unit vectors in the local toroidal coordinate system $\{r, \theta, \phi\}$ can now be determined; since $\widehat{\mathbf{r}}$ and $\widehat{\boldsymbol{\phi}}$ are orthogonal, we note that $\widehat{\mathbf{r}}=(\widehat{\mathbf{r}} \cdot \widehat{\mathbf{R}}) \widehat{\mathbf{R}}+(\widehat{\mathbf{r}} \cdot \hat{\mathbf{Z}}) \hat{\mathbf{Z}}$ and $\hat{\boldsymbol{\theta}}=\widehat{\boldsymbol{\phi}} \times \widehat{\mathbf{r}}$ so that $\widehat{\mathbf{r}}=\cos \theta \widehat{\mathbf{R}}+\sin \theta \hat{\mathbf{Z}}$ and $\hat{\boldsymbol{\theta}}=\cos \theta \hat{\mathbf{Z}}-\sin \theta \widehat{\mathbf{R}}$. In explicit form, we have

$$
\begin{aligned}
& \widehat{\mathbf{r}}=+\cos \theta \cos \phi \hat{\mathbf{x}}-\cos \theta \sin \phi \hat{\mathbf{y}}+\sin \theta \hat{\mathbf{z}} \\
& \hat{\boldsymbol{\theta}}=-\sin \theta \cos \phi \hat{\mathbf{x}}+\sin \theta \sin \phi \hat{\mathbf{y}}+\cos \theta \hat{\mathbf{z}} \\
& \hat{\boldsymbol{\phi}}=-\sin \phi \hat{\mathbf{x}}-\cos \phi \hat{\mathbf{y}} .
\end{aligned}
$$

The particle position vector, $\mathbf{r}$, can be written as $\mathbf{r}=\mathbf{R}_{\mathrm{gc}}+\boldsymbol{\rho}$, where $\mathbf{R}_{\mathrm{gc}}=\mathbf{R}_{\mathrm{gc}}\left(r_{0}, \theta_{0}, \phi_{0}\right)$ is the position of the guiding center and $\boldsymbol{\rho}=\widehat{\mathbf{e}}_{\|} \times \mathbf{v} / \omega_{\mathrm{c}}$ is the gyroradius vector; here $\widehat{\mathbf{e}}_{\|} \equiv \mathbf{B} / B$ is the unit vector along $\mathbf{B}$. The local unit vectors associated with $\mathbf{R}_{\mathrm{gc}}$ are similar to $\mathrm{Eq}(\mathrm{A} 1)$ with the replacement $\theta \mapsto \theta_{0}$ and $\phi \mapsto \phi_{0}$. Then we obtain

$$
\begin{aligned}
\widehat{\mathbf{r}} & =\left(\cos \theta \cos \theta_{0} \cos \Delta \phi+\sin \theta \sin \theta_{0}\right) \hat{\mathbf{r}}_{0}+\left(\sin \theta \cos \theta_{0}-\cos \theta \sin \theta_{0} \cos \Delta \phi\right) \hat{\boldsymbol{\theta}}_{0} \\
& +\cos \theta \sin \Delta \phi \widehat{\boldsymbol{\phi}}_{0} \\
\widehat{\boldsymbol{\theta}} & =\left(\cos \theta \sin \theta_{0}-\sin \theta \cos \theta_{0} \cos \Delta \phi\right) \widehat{\mathbf{r}}_{0}+\left(\cos \theta \cos \theta_{0}+\sin \theta \sin \theta_{0} \cos \Delta \phi\right) \hat{\boldsymbol{\theta}}_{0} \\
& -\sin \theta \sin \Delta \phi \widehat{\boldsymbol{\phi}}_{0} \\
\widehat{\boldsymbol{\phi}} & =-\cos \theta_{0} \sin \Delta \phi \hat{\mathbf{r}}_{0}+\sin \theta_{0} \sin \Delta \phi \hat{\boldsymbol{\theta}}_{0}+\cos \Delta \phi \hat{\boldsymbol{\phi}}_{0} .
\end{aligned}
$$

where $\Delta \phi \equiv \phi-\phi_{0}$. The particle velocity can be written as $\mathbf{v}=v_{\| \mid} \hat{\mathbf{e}}_{\|}+v_{\perp}(\cos \varphi \hat{\mathbf{n}}+\sin \varphi \hat{\mathbf{b}})$, where $\hat{\mathbf{n}} \equiv\left[\nabla \Psi /(\nabla \Psi \cdot \nabla \Psi)^{1 / 2}\right]_{r_{0}, \theta_{0}, \zeta_{0}}$ is the unit normal vector, $\hat{\mathbf{e}}_{\|} \equiv \mathbf{B} / B$ is the unit vector along $\mathbf{B}, \hat{\mathbf{b}} \equiv\left[\hat{\mathbf{e}}_{\|} \times \hat{\mathbf{n}}\right]_{r_{0}, \theta_{0}, \zeta_{0}}$ is the unit binormal vector, and $\varphi$ is the gyroangle (Fig. 2). 
Then we may write the gyroradius vector as $\rho=\omega_{c}^{-1}\left(\widehat{\mathbf{e}}_{||} \times \mathbf{v}\right)=\rho(\cos \varphi \widehat{\mathbf{b}}-\sin \varphi \widehat{\mathbf{n}})$, where $\rho \equiv v_{\perp} / \omega_{c}$. Without loss of generality, the relation $\mathbf{r}=\mathbf{R}_{\mathrm{gc}}+\boldsymbol{\rho}$ can be written as

$$
R_{0} \widehat{\mathbf{R}}+r \widehat{\mathbf{r}}=R_{0} \widehat{\mathbf{R}}_{g c}+r_{0} \widehat{\mathbf{r}}_{0}+\boldsymbol{\rho}
$$

where $\widehat{\mathbf{R}}_{\mathrm{gc}} \equiv \cos \phi_{0} \widehat{\mathbf{x}}-\sin \phi_{0} \hat{\mathbf{y}}$. Taking the scalar product of equation (A3) with $\widehat{\mathbf{r}}_{0}, \widehat{\boldsymbol{\theta}}_{0}$ and $\widehat{\boldsymbol{\phi}}_{0}$, noting that $\widehat{\mathbf{R}}_{\mathrm{gc}} \cdot \widehat{\boldsymbol{\phi}}_{0}=\widehat{\mathbf{r}}_{0} \cdot \widehat{\boldsymbol{\phi}}_{0}=0, \widehat{\mathbf{R}} \cdot \widehat{\phi}_{0}=\cos \theta \hat{\mathbf{r}} \cdot \widehat{\boldsymbol{\phi}}_{0}-\sin \theta \hat{\boldsymbol{\theta}} \cdot \widehat{\boldsymbol{\phi}}_{0}=\sin \Delta \phi, \widehat{\mathbf{R}} \cdot \widehat{\mathbf{r}}_{0}=$ $\cos \theta \hat{\mathbf{r}} \cdot \widehat{\mathbf{r}}_{0}-\sin \theta \hat{\boldsymbol{\theta}} \cdot \widehat{\mathbf{r}}_{0}=\cos \theta_{0} \cos \Delta \phi, \widehat{\mathbf{R}} \cdot \hat{\boldsymbol{\theta}}_{0}=\cos \theta \hat{\mathbf{r}} \cdot \widehat{\boldsymbol{\theta}}_{0}-\sin \theta \hat{\boldsymbol{\theta}} \cdot \hat{\boldsymbol{\theta}}_{0}=-\sin \theta_{0} \cos \Delta \phi$, neglecting corrections $\mathcal{O}\left(\rho^{2} / R_{0}^{2}\right)$ and higher we get

$$
\begin{aligned}
\sin \Delta \phi & =\frac{\boldsymbol{\rho} \cdot \widehat{\boldsymbol{\phi}}_{0}}{R} \\
r \cos \left(\theta-\theta_{0}\right)-r_{0} & =\boldsymbol{\rho} \cdot \widehat{\mathbf{r}}_{0} \\
r \sin \left(\theta-\theta_{0}\right) & =\boldsymbol{\rho} \cdot \widehat{\boldsymbol{\theta}}_{0} .
\end{aligned}
$$

The geometrical effects are contained in the $\boldsymbol{\rho} \cdot \widehat{\boldsymbol{r}}_{0}, \boldsymbol{\rho} \cdot \widehat{\boldsymbol{\theta}}_{0}$ and $\boldsymbol{\rho} \cdot \widehat{\boldsymbol{\phi}}_{0}$ terms. Introducing a new gyroangle $\psi \equiv \varphi-3 \pi / 2$, we obtain the following set of equations

$$
\begin{aligned}
& r \cos \Delta \theta-\rho \cos \psi\left(\widehat{\mathbf{n}} \cdot \hat{\mathbf{r}}_{0}\right)=r_{0}+\rho \sin \psi\left(\hat{\mathbf{b}} \cdot \hat{\mathbf{r}}_{0}\right) \\
& r \sin \Delta \theta-\rho \sin \psi\left(\widehat{\mathbf{b}} \cdot \hat{\boldsymbol{\theta}}_{0}\right)=\rho \cos \psi\left(\hat{\mathbf{n}} \cdot \widehat{\boldsymbol{\theta}}_{0}\right)
\end{aligned}
$$

It is convenient to introduce new variables $\rho$ " and $\psi^{\prime \prime}$ such that

$$
\begin{aligned}
\rho^{\prime \prime} \sin \psi^{\prime \prime} & =\rho \sin \psi\left(\hat{\mathbf{b}} \cdot \hat{\boldsymbol{\theta}}_{0}\right)+\rho \cos \psi\left(\hat{\mathbf{n}} \cdot \hat{\boldsymbol{\theta}}_{0}\right) \\
\rho^{\prime \prime} \cos \psi^{\prime \prime} & =\rho \cos \psi\left(\hat{\mathbf{n}} \cdot \hat{\mathbf{r}}_{0}\right)+\rho \sin \psi\left(\widehat{\mathbf{b}} \cdot \hat{\mathbf{r}}_{0}\right)
\end{aligned}
$$

Then we may re-write equations (A5) in a simpler form

$$
\begin{aligned}
& r \cos \Delta \theta-\rho^{\prime \prime} \cos \psi^{\prime \prime}=r_{0} \\
& r \sin \Delta \theta-\rho^{\prime \prime} \sin \psi^{\prime \prime}=0 .
\end{aligned}
$$

Using (A4) and $\psi=\varphi-3 \pi / 2$, we note that $\Delta \phi \simeq \rho\left[\cos \psi\left(\widehat{\mathbf{n}} \cdot \hat{\boldsymbol{\phi}}_{0}\right)+\sin \psi\left(\hat{\mathbf{b}} \cdot \hat{\boldsymbol{\phi}}_{0}\right)\right] / R$; in a tokamak with $B_{\theta} \ll B_{\phi}$, one gets $\hat{\mathbf{b}} \mapsto \hat{\boldsymbol{\theta}}_{0}$ so that $\Delta \phi \mapsto 0$ and the problem is essentially two-dimensional. To leading order, one can show that 


$$
\begin{aligned}
& \Delta \theta \simeq \frac{\rho^{\prime \prime}}{r_{0}} \sin \psi^{\prime \prime} \\
& \Delta \phi \simeq f \frac{\rho^{\prime \prime}}{R} \cos \psi^{\prime \prime}+g \frac{\rho^{\prime \prime}}{R} \sin \psi^{\prime \prime},
\end{aligned}
$$

where

$$
f \equiv \frac{\widehat{\mathbf{n}} \cdot \hat{\phi}_{0}}{\hat{\mathbf{n}} \cdot \hat{\mathbf{r}}_{0}} \text { and } g \equiv \frac{\hat{\mathbf{b}} \cdot \hat{\phi}_{0}}{\widehat{\mathbf{b}} \cdot \widehat{\boldsymbol{\theta}}_{0}}
$$

In a low- $\beta$ tokamak plasma with circular magnetic surfaces, $f=0$ and $g \sim B_{\theta} / B_{\phi}=\mathcal{O}(\epsilon) \ll$ 1 , where $\epsilon$ is the inverse aspect ratio. It is easy to see that $f$ is related to the non-circularity of the magnetic surfaces; in general, we expect $f \ll g$ (at least in the average sense). In the complex plane, equations $(A 7)$ can be written in compact form as

$$
r \exp (i \theta)=\rho^{\prime \prime} \exp \left(i \psi^{\prime \prime}+i \theta_{0}\right)+r_{0} \exp \left(i \theta_{0}\right)
$$

For an arbitrary function $F$, we define $\langle F\rangle_{\mathrm{gc}}=(2 \pi)^{-1} \int_{-\pi}^{+\pi} F(r, \theta, \phi, \varphi) d \varphi$ (for fixed guiding center position) and $\langle F\rangle_{\text {part }}=(2 \pi)^{-1} \int_{-\pi}^{+\pi} F\left(r_{0}, \theta_{0}, \phi_{0}, \varphi\right) d \varphi$ (for fixed particle position). For fixed guiding center (fixed $\mathrm{r}_{0}, \theta_{0}, \phi_{0}$ ), one can use Graf's theorem ${ }^{15}$

$$
J_{N}(w) \exp (i N \theta)=\sum_{k=-\infty}^{\infty} J_{N+k}(u) J_{k}(v) \exp (i k \alpha)
$$

where $u, v, w$ and $\alpha$ satisfy $w=\left(u^{2}+v^{2}-2 u v \cos \alpha\right)^{1 / 2}, u-v \cos \alpha=w \cos \theta, v \sin \alpha=$ $w \sin \theta$, to get

$$
J_{m}\left(\beta_{m l} r\right) \exp \left[i m\left(\theta-\theta_{0}\right)\right]=\sum_{k=-\infty}^{\infty} J_{m+k}\left(\beta_{m l} r_{0}\right) J_{k}\left(\beta_{m l} \rho^{\prime \prime}\right) \exp \left[-i k\left(\pi+\psi^{\prime \prime}\right)\right] .
$$

Similarly, for fixed particle position, Graf's theorem (A11) yields

$$
J_{m}\left(\beta_{m l} r_{0}\right) \exp \left[i m\left(\theta_{0}-\theta\right)\right]=\sum_{k=-\infty}^{\infty} J_{m+k}\left(\beta_{m l} r\right) J_{k}\left(\beta_{m l} \rho^{\prime \prime}\right) \exp \left[i k\left(\theta-\theta_{0}-\psi^{\prime \prime}\right)\right] .
$$

We multiply equation $(\mathrm{A} 12)$ by $\exp \left(i n \phi+i m \theta_{0}\right)=\exp \left(i n \phi_{0}+i m \theta_{0}\right) \exp (i n \Delta \phi)$ and operate with $\langle\ldots\rangle_{\mathrm{gc}}$ on the resulting equation

$$
\begin{aligned}
\left\langle J_{m}\left(\beta_{m l} r\right) \exp (i m \theta+i n \phi)\right\rangle_{\mathrm{gc}} & =\exp \left(i m \theta_{0}+i n \phi_{0}\right) \sum_{k=-\infty}^{\infty} J_{m+k}\left(\beta_{m l} r_{0}\right) J_{k}\left(\beta_{m l} \rho^{\prime \prime}\right) \\
& \times\left\langle\exp (i n \Delta \phi) \exp \left[-i k\left(\pi+\psi^{\prime \prime}\right)\right]\right\rangle_{\mathrm{gc}}
\end{aligned}
$$


Taking into account $|f| \ll|g|$, we may write in $\Delta \phi \approx k_{\mathrm{t}} \rho^{\prime \prime} \sin \psi^{\prime \prime}$; here $k_{\mathrm{t}} \equiv n g / R$ is the toroidal wavenumber where, as before, $R=R_{0}+r \cos \theta$ is the cylindrical radius. Then

$$
\begin{aligned}
\left\langle J_{m}\left(\beta_{m l} r\right) \exp (i m \theta+i n \phi)\right\rangle_{\mathrm{gc}} & =\exp \left(i m \theta_{0}+i n \phi_{0}\right) \sum_{k=-\infty}^{\infty} J_{m+k}\left(\beta_{m l} r_{0}\right) J_{k}\left(\beta_{m l} \rho^{\prime \prime}\right) \exp (-i k \pi) \\
& \times\left\langle\exp \left(i k_{\mathrm{t}} \rho^{\prime \prime} \sin \psi^{\prime \prime}-i k \psi^{\prime \prime}\right)\right\rangle_{\mathrm{gc}} \\
& =\exp \left(i m \theta_{0}+i n \phi_{0}\right) \sum_{k=-\infty}^{\infty}(-1)^{k} J_{m+k}\left(\beta_{m l} r_{0}\right) J_{k}\left(\beta_{m l} \rho^{\prime \prime}\right) J_{k}\left(k_{\mathrm{t}} \rho^{\prime \prime}\right) \\
& =\exp (i m \theta+i n \phi)[\exp (-i m \Delta \theta) \exp (-i n \Delta \phi) \\
& \left.\times \sum_{k=-\infty}^{\infty}(-1)^{k} J_{m+k}\left(\beta_{m l} r_{0}\right) J_{k}\left(\beta_{m l} \rho^{\prime \prime}\right) J_{k}\left(k_{\mathrm{t}} \rho^{\prime \prime}\right)\right]
\end{aligned}
$$

where we used the integral representation for Bessel functions ${ }^{14}$

$$
J_{N}(x)=\frac{1}{\pi} \int_{0}^{\pi} \cos (x \sin \theta-N \theta) d \theta=\frac{i^{-N}}{\pi} \int_{0}^{\pi} \exp (i x \cos \theta) \cos (N \theta) d \theta .
$$

Using the recurrence relation ${ }^{14}, d J_{N} / d x=\left[J_{N-1}(x)-J_{N+1}(x)\right] / 2$ (for $N$ integer), we may write and

$$
J_{m+k}\left(\beta_{m l} r_{0}\right)=J_{m+k}\left(\beta_{m l} r\right)+\frac{\beta_{m l}}{2} \rho^{\prime \prime} \cos \lambda\left[J_{m+k+1}\left(\beta_{m l} r\right)-J_{m+k-1}\left(\beta_{m l} r\right)\right]+\ldots
$$

in equation (A15); operating with $\langle\ldots\rangle_{\text {part }}$ (keeping $r, \theta$ and $\phi$ constants) on the resulting equation, one gets

$$
\begin{aligned}
\left\langle\left\langle J_{m}\left(\beta_{m l} r\right) \exp (i m \theta+i n \phi)\right\rangle_{\mathrm{gc}}\right\rangle_{\mathrm{part}} & =\exp (i m \theta+i n \phi)\left\{\sum_{k=-\infty}^{\infty}(-1)^{k} J_{k}\left(\beta_{m l} \rho^{\prime \prime}\right) J_{k}\left(k_{\mathrm{t}} \rho^{\prime \prime}\right)\right. \\
& \times J_{m+k}\left(\beta_{m l} r\right)\langle\exp (-i m \Delta \theta-i n \Delta \phi)\rangle_{\mathrm{part}} \\
& +\frac{\beta_{m l}}{2} \rho^{\prime \prime} \sum_{k=-\infty}^{\infty}(-1)^{k} J_{k}\left(\beta_{m l} \rho^{\prime \prime}\right) J_{k}\left(k_{\mathrm{t}} \rho^{\prime \prime}\right) \\
& \times\left[J_{m+k+1}\left(\beta_{m l} r\right)-J_{m+k-1}\left(\beta_{m l} r\right)\right] \\
& \left.\times\langle\cos \lambda \exp (-i m \Delta \theta-i n \Delta \phi)\rangle_{\mathrm{part}}+\ldots\right\}
\end{aligned}
$$

where $\lambda=\Delta \theta-\psi^{\prime \prime}$ and $m \Delta \theta \approx k_{\mathrm{p}} \rho^{\prime \prime} \sin \psi^{\prime \prime}$, where $k_{\mathrm{p}} \equiv m / r$ is the poloidal wavenumber. Let $\hat{k} \equiv k_{\mathrm{p}}+k_{\mathrm{t}}$ and use the integral representation (A16) to show that $\langle\exp (-i m \Delta \theta-i n \Delta \phi)\rangle_{\text {part }}=J_{0}\left(\hat{k} \rho^{\prime \prime}\right)$. Write $\cos \lambda=[\exp (i \lambda)+\exp (-i \lambda)] / 2$ to obtain $\langle\cos \lambda \exp (-i m \Delta \theta-i n \Delta \phi)\rangle_{\text {part }}$ 
$\left\langle\exp \left(-i x_{-} \sin \psi^{\prime \prime}-i \psi^{\prime \prime}\right)\right\rangle_{\text {part }} / 2+\left\langle\exp \left(-i x_{+} \sin \psi^{\prime \prime}+i \psi^{\prime \prime}\right)\right\rangle_{\text {part }} / 2=\left[J_{1}\left(x_{+}\right)-J_{1}\left(x_{-}\right)\right] / 2$, where $x_{ \pm} \equiv(\hat{k} \pm 1 / r) \rho^{\prime \prime}$. Combining the above results we can write

$$
\begin{array}{r}
\left\langle\left\langle J_{m}\left(\beta_{m l} r\right) \exp (i m \theta+i n \phi)\right\rangle_{\mathrm{gc}}\right\rangle_{\mathrm{part}} \\
\quad=\exp (i m \theta+i n \phi)\left\{\sum_{k=-\infty}^{\infty}(-1)^{k} J_{k}\left(\beta_{m l} \rho^{\prime \prime}\right) J_{k}\left(k_{\mathrm{t}} \rho^{\prime \prime}\right)\right. \\
\times J_{m+k}\left(\beta_{m l} r\right) J_{0}\left(\hat{k} \rho^{\prime \prime}\right)+\frac{\beta_{m l}}{4} \rho^{\prime \prime} \sum_{k=-\infty}^{\infty}(-1)^{k} J_{k}\left(\beta_{m l} \rho^{\prime \prime}\right) J_{k}\left(k_{\mathrm{t}} \rho^{\prime \prime}\right) \\
\left.\times\left[J_{m+k+1}\left(\beta_{m l} r\right)-J_{m+k-1}\left(\beta_{m l} r\right)\right]\left[J_{1}\left(x_{+}\right)-J_{1}\left(x_{-}\right)\right]\right\}
\end{array}
$$

For the case of a strong toroidal magnetic field, the above result [Eq (A19)] can be considerably simplified. In the limit $B_{\theta} \mapsto 0$, we note that $\hat{\mathbf{e}}_{\|}=\mathbf{B} / B \mapsto \hat{\boldsymbol{\phi}}_{0}, \hat{\mathbf{b}} \mapsto \hat{\boldsymbol{\theta}}_{0}$ and $\Delta \phi=\phi-\phi_{0} \mapsto 0$. Using equation (A12), we have

$$
\left\langle J_{m}\left(\beta_{m l} r\right) \exp (i m \theta+i n \phi)\right\rangle_{\mathrm{gc}}=\exp \left(i m \theta_{0}+i n \phi\right) J_{m}\left(\beta_{m l} r_{0}\right) J_{0}\left(\beta_{m l} \rho^{\prime \prime}\right)
$$

since $\left\langle\exp \left[-i k\left(\pi+\psi^{\prime \prime}\right)\right]\right\rangle_{\mathrm{gc}}=\delta(k)$. Operating with $\langle\ldots\rangle_{\mathrm{part}}$ on equation (A20) and using equation (A13), we get

$$
\begin{aligned}
\left\langle\left\langle J_{m}\left(\beta_{m l} r\right) \exp (i m \theta+i n \phi)\right\rangle_{\mathrm{gc}}\right\rangle_{\mathrm{part}} & =\exp (i n \phi)\left\langle\exp \left(i m \theta_{0}\right) J_{m}\left(\beta_{m l} r_{0}\right)\right\rangle_{\mathrm{part}} J_{0}\left(\beta_{m l} \rho^{\prime \prime}\right) \\
& =\exp (i m \theta+i n \phi) \\
& \times\left\langle\sum_{k=-\infty}^{\infty} J_{m+k}\left(\beta_{m l} r\right) J_{k}\left(\beta_{m l} \rho^{\prime \prime}\right) \exp \left[i k\left(\theta-\theta_{0}-\psi^{\prime \prime}\right)\right]\right\rangle_{\mathrm{part}} J_{0}\left(\beta_{m l} \rho^{\prime \prime}\right) \\
& =\exp (i m \theta+i n \phi) \sum_{k=-\infty}^{\infty} J_{m+k}\left(\beta_{m l} r\right) J_{k}\left(\beta_{m l \rho} \rho^{\prime \prime}\right) J_{0}\left(\beta_{m l \rho} \rho^{\prime \prime}\right) \\
& \times\left\langle\exp \left[i k\left(\theta-\theta_{0}-\psi^{\prime \prime}\right)\right]\right\rangle_{\mathrm{part}} \\
& =\exp (i m \theta+i n \phi) J_{m}\left(\beta_{m l} r\right) J_{0}{ }^{2}\left(\beta_{m l \rho}{ }^{\prime \prime}\right)
\end{aligned}
$$

which is the expression derived by Li et al. 


\section{APPENDIX B: FLUX-SURFACE AVERAGE OF THE GKP EQUATION}

In the local toroidal system $\{r, \theta, \phi\}$, the surface average of $F$ is

$$
\langle F\rangle_{S} \equiv \frac{1}{A} \int_{0}^{2 \pi} d \phi \int_{0}^{2 \pi} d \theta F(r, \theta, \phi) \mathcal{J}(r, \theta)
$$

where $\mathcal{J}=[\nabla r \cdot(\nabla \theta \times \nabla \phi)]^{-1}=r\left(R_{0}+r \cos \theta\right)$ is the Jacobian of the transformation and $A \equiv \int_{0}^{2 \pi} d \phi \int_{0}^{2 \pi} d \theta \mathcal{J}(r, \theta)=4 \pi^{2} r R_{0}$ is the area of the magnetic surface. Then we note that

$$
\langle\exp (i m \theta+i n \phi)\rangle_{S}=\delta(n) \delta(m)+\frac{r}{2 R_{0}} \delta(n)[\delta(m+1)+\delta(m-1)],
$$

where the second term on the right-hand side is a finite-toroidicity correction. The surface average of $\langle\tilde{\Phi}\rangle_{\text {part }}$ involves terms of the form $\left\langle J_{k}(F) J_{l}(G) J_{m}(H) \exp (\operatorname{im} \theta+i n \phi)\right\rangle_{\text {part }}$, where, in general, $F=F(r, \theta, \phi), G=G(r, \theta, \phi), H=H(r, \theta, \phi)$, and $k, l, m$ are integers. Without loss of generality, we can write

$$
F(r, \theta, \phi)=\bar{F}(1-f)
$$

where $\bar{F}(r) \equiv\langle F\rangle_{S}$ is a flux surface quantity, and $f(r, \theta, \phi) \equiv 1-F(r, \theta, \phi) / \bar{F}$. Similarly one may write $G=\bar{G}(1-g)$ and $H=\bar{H}(1-h)$; by construction, we note that $\langle f\rangle_{S}=$ $\langle g\rangle_{S}=\langle h\rangle_{S}=0$. Typically the ratio $|f / \bar{F}|$ is of the order of the inverse aspect ratio. Using a Taylor expansion

$$
J_{k}(F)=J_{k}(\bar{F})+\frac{f}{2}\left[J_{k+1}(\bar{F})-J_{k-1}(\bar{F})\right]+\ldots
$$

and similarly for $J_{l}(G)$ and $J_{m}(H)$, we obtain (neglecting higher-order corrections)

$$
\begin{aligned}
\left\langle J_{k}(F) J_{l}(G)\right. & \left.J_{m}(H) \exp (i m \theta+i n \phi)\right\rangle_{S}=J_{k}(\bar{F}) J_{l}(\bar{G}) J_{m}(\bar{H})\langle\exp (i m \theta+i n \phi)\rangle_{S} \\
& +\frac{1}{2} J_{k}(\bar{F}) J_{l}(\bar{G})\left[J_{m+1}(\bar{H})-J_{m-1}(\bar{H})\right]\langle h(r, \theta, \phi) \exp (i m \theta+i n \phi)\rangle_{S} \\
& \left.+\frac{1}{2} J_{k}(\bar{F}) J_{m}(\bar{H})\left[J_{l+1}(\bar{G})-J_{l-1}(\bar{G})\right)\right]\langle g(r, \theta, \phi) \exp (i m \theta+i n \phi)\rangle_{S} \\
+ & \left.\frac{1}{2} J_{l}(\bar{G}) J_{m}(\bar{H})\left[J_{k+1}(\bar{F})-J_{k-1}(\bar{F})\right)\right]\langle f(r, \theta, \phi) \exp (i m \theta+i n \phi)\rangle_{S}+\ldots
\end{aligned}
$$

Then, to leading order, the flux-surface average of $\langle\tilde{\Phi}\rangle_{\text {part }}$ becomes 


$$
\begin{array}{r}
\left\langle\langle\tilde{\Phi}\rangle_{\text {part }}\right\rangle_{S}=\sum_{l=1}^{\infty} \sum_{m=-\infty}^{\infty} \sum_{n=-\infty}^{\infty} \Phi_{l m n}\left\{J_{m}\left(\beta_{m l} r\right)-\sum_{k=-\infty}^{\infty}(-1)^{k} J_{k}\left[\beta_{m l}\left\langle\rho_{\mathbf{i}}\right\rangle_{S} V_{\perp}\right] J_{k}\left[\left\langle k_{t} \rho_{\mathbf{i}}\right\rangle_{S} V_{\perp}\right]\right. \\
J_{m+k}\left(\beta_{m l l} r\right) J_{0}\left[\left[\left\langle\hat{k} \rho_{\mathbf{i}}\right\rangle_{S} V_{\perp}\right]+\frac{\beta_{m l}}{4}\left\langle\rho_{\mathbf{i}}\right\rangle_{S} V_{\perp} \sum_{k=-\infty}^{\infty}(-1)^{k} J_{k}\left[\beta_{m l}\left\langle\rho_{\mathrm{i}}\right\rangle_{S} V_{\perp}\right] J_{k}\left[\left\langle k_{\mathrm{t}} \rho_{\mathrm{i}}\right\rangle_{S} V_{\perp}\right]\right. \\
\left.\left[J_{m+k+1}\left(\beta_{m l} r\right)-J_{m+k-1}\left(\beta_{m l} r\right)\right]\left[J_{1}\left(y_{+} V_{\perp}\right)-J_{1}\left(y_{-} V_{\perp}\right)\right]+\ldots\right\}\langle\exp (i m \theta+i n \phi)\rangle_{S}
\end{array}
$$

where $y_{ \pm} \equiv\left[(m \pm 1) / r+n / R_{0}\right]\left\langle\rho_{\mathbf{i}}\right\rangle_{S}$. Here $\left\langle\rho_{\mathbf{i}}\right\rangle_{S}$ is the surface-average of the ion thermal gyroradius $\rho_{\mathrm{i}} \equiv v_{\mathrm{thi}} / \omega_{\mathrm{c}}$. Since the toroidal wavenumber $k_{\mathrm{t}} \propto 1 / R$, we note that $\left\langle k_{\mathrm{t}} \rho_{\mathrm{i}}\right\rangle_{S} \neq$ $k_{t}\left\langle\rho_{\mathbf{i}}\right\rangle_{S}$. Equation (B6) can be simplified by using the following properties ${ }^{14}$ of the Bessel functions $J_{N}(x) \sim(x / 2)^{N} / \Gamma(N+1)$ for $|x| \mapsto 0$, and $J_{-N}(x)=(-1)^{N} J_{N}(x)$; whereas the integral representation (A16) shows that $J_{k}(0)=\delta(k)$. We obtain

$$
\left\langle\langle\tilde{\Phi}\rangle_{\text {part }}\right\rangle_{S}=\sum_{l=1}^{\infty} \Phi_{l 00}\left[J_{0}\left(\beta_{0 l} r\right)-J_{0}\left(\beta_{0 l}\left\langle\rho_{\mathbf{i}}\right\rangle_{S} V_{\perp}\right) J_{0}\left(\beta_{0 l} r\right)+\frac{\beta_{0 l}}{2} \frac{\bar{\rho}^{2} / r}{\Gamma(2)} V_{\perp}^{2} J_{1}\left(\beta_{0 l} r\right) J_{0}\left(\beta_{0 l}\left\langle\rho_{\mathbf{i}}\right\rangle_{S} V_{\perp}\right)+\ldots\right]
$$

Substituting equation (B7) in the GKP equation (3) we get

$$
\begin{array}{r}
\sum_{l=1}^{\infty} \Phi_{l 00}\left[J_{0}\left(\beta_{0 l} r\right)-J_{0}\left(\beta_{0 l} r\right)\left[\int_{0}^{\infty} V_{\perp} \exp \left(-V_{\perp}^{2} / 2\right) J_{0}\left(\beta_{0 l}\left\langle\rho_{\mathrm{i}}\right\rangle_{S} V_{\perp}\right) d V_{\perp}\right]\right. \\
\left.+\frac{\beta_{0 l}}{2} \frac{\left\langle\rho_{\mathrm{i}}\right\rangle_{S}{ }^{2} / r}{\Gamma(2)} J_{1}\left(\beta_{0 l} r\right)\left[\int_{0}^{\infty} V_{\perp}^{3} \exp \left(-V_{\perp}^{2} / 2\right) J_{0}\left(\beta_{0 l}\left\langle\rho_{\mathrm{i}}\right\rangle_{S} V_{\perp}\right) d V_{\perp}\right]+\ldots\right]=\frac{T_{\mathrm{i} 0}}{e n_{0}}\left\langle\overline{n_{\mathrm{i}}}-n_{\mathrm{e}}^{(\mathrm{NA})}\right\rangle_{S}
\end{array}
$$

The first integral on the left-hand side of equation (B8) is of the form ${ }^{15}$

$$
\mathcal{L}_{\nu}(\alpha, \beta)=\int_{0}^{\infty} x^{\nu+1} \exp \left(-\alpha x^{2}\right) J_{\nu}(\beta x) d x=\frac{\beta^{\nu}}{(2 \alpha)^{\nu+1}} \exp \left(-\frac{\beta^{2}}{4 \alpha}\right) .
$$

Noting that

$$
\int_{0}^{\infty} x^{3} \exp \left(-\alpha x^{2}\right) J_{0}(\beta x) d x=-\frac{\partial \mathcal{L}_{0}(\alpha, \beta)}{\partial \alpha}=\frac{1}{2 \alpha^{2}}\left(1-\frac{\beta^{2}}{4 \alpha}\right) \exp \left(-\frac{\beta^{2}}{4 \alpha}\right),
$$

we obtain an equation governing the sheared-flow modes in toroidal geometry

$\sum_{l=1}^{\infty} \Phi_{l 00}\left\{J_{0}\left(\beta_{0 l} r\right)\left[1-\exp \left(-C_{l}^{2} / 2\right)\right]+\frac{\left(\left\langle\rho_{\mathbf{i}}\right\rangle_{S} / r\right)}{\Gamma(2)} J_{1}\left(\beta_{0 l} r\right) C_{l}\left(1-\frac{C_{l}^{2}}{2}\right) \exp \left(-C_{l}^{2} / 2\right)\right\}=\mathcal{D}(r)$,

where $C_{l} \equiv \beta_{0 l}\left\langle p_{\mathrm{i}}\right\rangle_{S}$ and $\mathcal{D}(r) \equiv T_{\mathrm{i} 0}\left[\left\langle\overline{n_{\mathrm{i}}}\right\rangle_{S}-\left\langle n_{\mathrm{e}}^{(\mathrm{NA})}\right\rangle_{S}\right] /\left(e n_{0}\right)$. 


\section{REFERENCES}

${ }^{1}$ W. M. Tang, Nucl. Fusion 18, 1089 (1978).

${ }^{2}$ W. Horton, Rev. Mod. Phys. 71, 735 (1999).

${ }^{3}$ P. C. Liewer, Nucl. Fusion 25, 543 (1985).

${ }^{4}$ F. Wagner and U. Stroth, Plasma Phys. Contr. Fusion, 35, 1321 (1993).

${ }^{5}$ K.H. Burrell, Phys. Plasmas 4, 1499 (1997).

${ }^{6}$ P.H. Diamond, M.N. Rosenbluth, F.L. Hinton et al in Proceedings of the 1 ith IAEA Conference on Controlled Fusion and Plasma Physics (Yokahama, Japan, 1997).

${ }^{7}$ T.S. Hahm and K.H. Burrell, Phys. Plasmas 2, 1648 (1995).

${ }^{8}$ W.W. Lee, Phys. Fluids 26, 556 (1983)

${ }^{9}$ Z. Lin and W.W. Lee, Phys. Rev. E 52, 5646 (1995)

${ }^{10}$ W.W. Lee, J. Comput. Phys. 72, 243 (1987)

11 T.S. Hahm, Phys. Fluids 31, 2670 (1988)

${ }^{12}$ S.P. Hirshman and J.C. Whitson, Phys. Fluids 26, 3553 (1983).

${ }^{13}$ S.P. Hirshman and H.K. Meier, Phys. Fluids 28, 1387 (1985).

${ }^{14}$ M. Abramowitz and I.A. Stegun, Handbook of Mathematical Functions, (National Bureau of Standards, Washington, 1965).

${ }^{15}$ I.S. Gradshteyn and I.M. Ryzhik, Table of Integrals, Series, and Products, (Academic Press, New York, 1965).

${ }^{16}$ P.H. Diamond and Y.-B. Kim, Phys. Fluids B 3(7), 1626 (1991). 
Figure 1 Cylindrical coordinate system $(R, Z, \phi)$ and local toroidal coordinate system $\left(r_{L}, \theta_{L}, \phi_{\mathrm{L}} \equiv \phi\right)$ in toroidal geometry. A magnetic surface $\bar{\rho}=$ const (plain line) and a tokamak-like magnetic surface (dotted line) are shown.

Figure 2 Using the local coordinate system (Figure 1), the particle position, r, and its guiding center position, $\mathbf{R}_{\mathrm{gc}}$, can be labeled by $(r, \theta, \phi)$ and $\left(R_{\mathrm{gc}}, \theta_{0}, \phi_{0}\right)$, respectively. Here $r \equiv(\mathbf{r} \cdot \mathbf{r})^{1 / 2}$ and $R_{\mathrm{gc}} \equiv\left(\mathbf{R}_{\mathrm{gc}} \cdot \mathbf{R}_{\mathrm{gc}}\right)^{1 / 2}$.

Figure 3 The perpendicular component of the particle velocity can be written as $\mathbf{v}_{\perp}=$ $v_{\perp}(\cos \varphi \hat{\mathbf{n}}+\sin \varphi \hat{\mathbf{b}})$, where $\hat{\mathbf{n}}$ is normal to the magnetic surface, and $\hat{\mathbf{b}} \propto \mathbf{B} \times \hat{\mathbf{n}}$ is the binormal vector. The gyrradius vector, $\boldsymbol{\rho}$, is defined as $\boldsymbol{\rho} \equiv(\mathbf{B} / B) \times \mathbf{v} / \omega_{\mathrm{c}}$ where $\omega_{\mathrm{c}}$ is the cyclotron frequency. 
FIG.1 Lewandowski et al

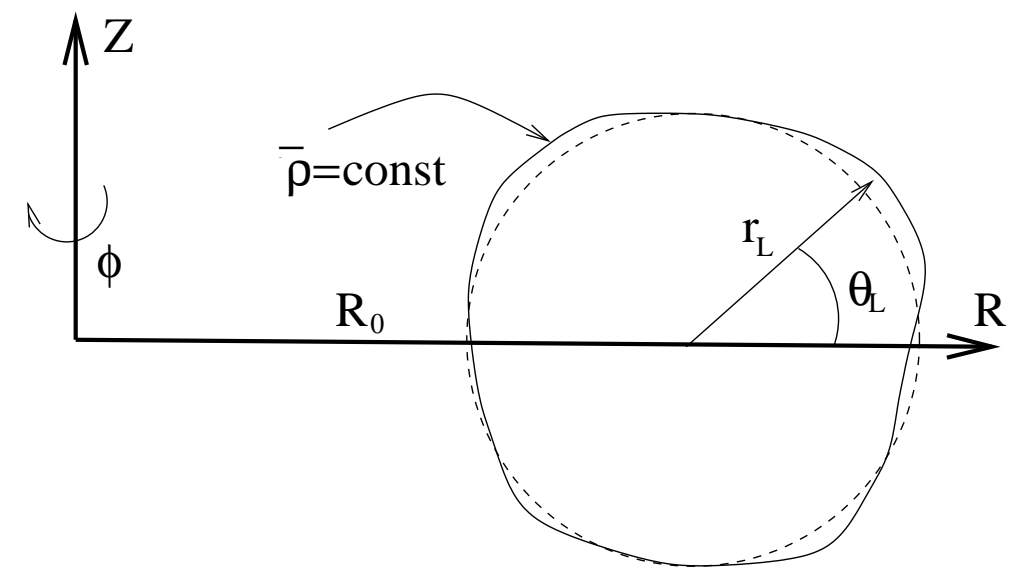


FIG.2 Lewandowski et al

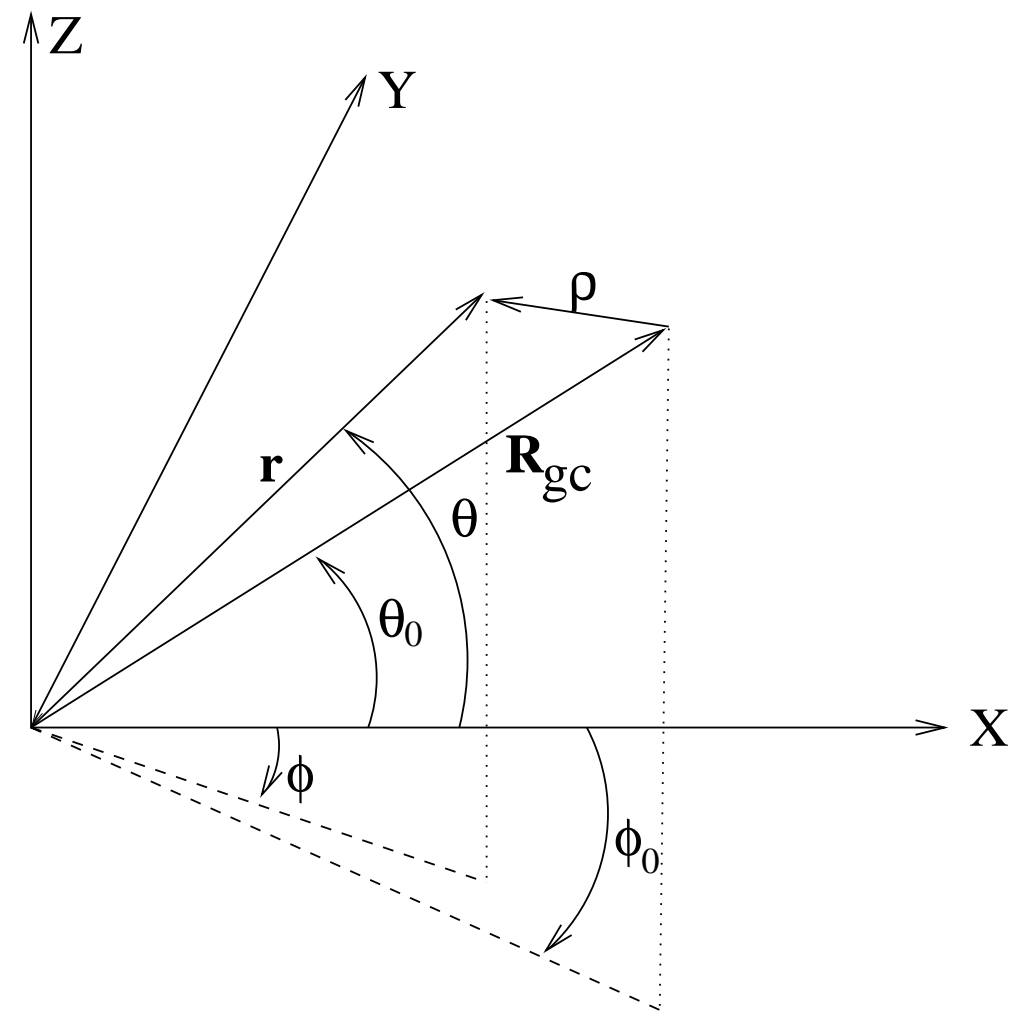


FIG.3 Lewandowski et al

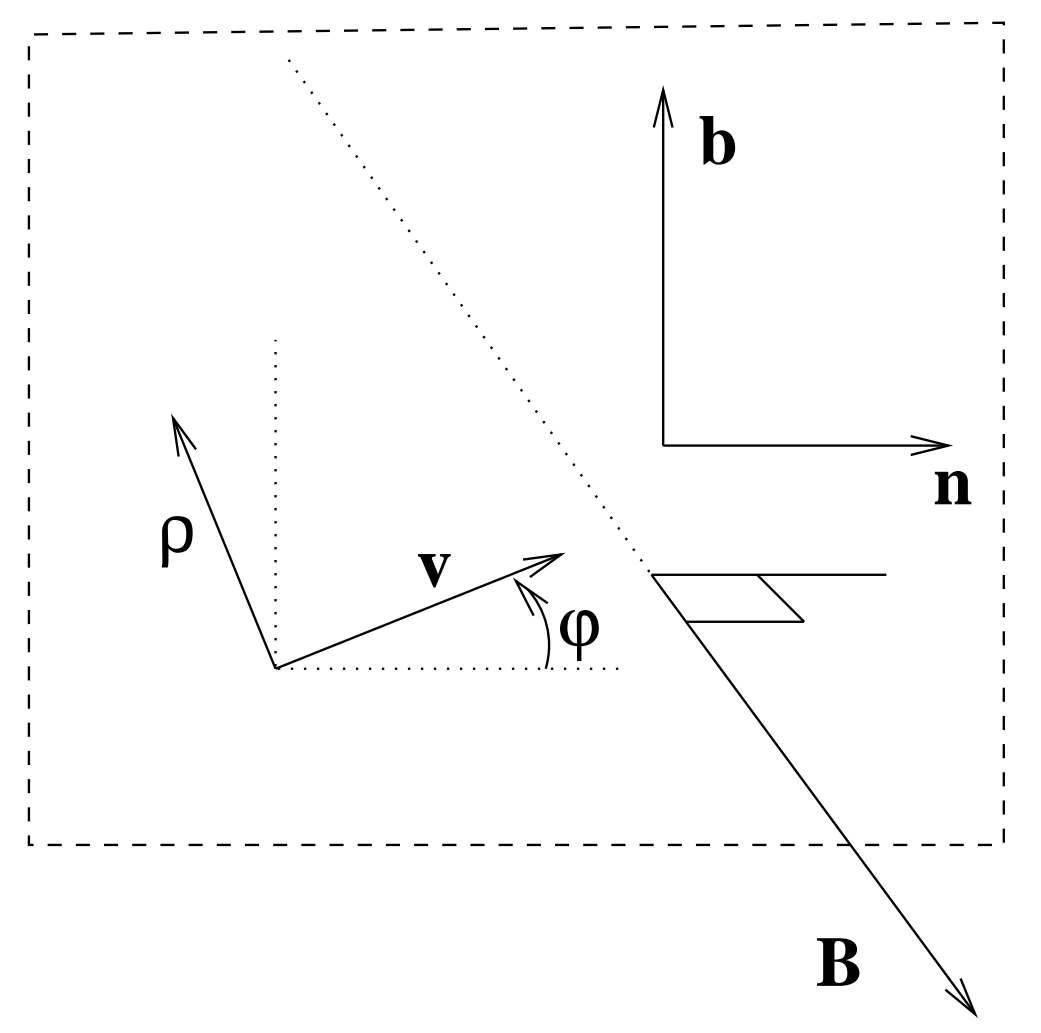

